DOI : $10.24850 /$ j-tyca-2021-04-10

Artículos

\title{
Determinación del coeficiente de avance de la tormenta según el origen y peligro de las precipitaciones en el archipiélago cubano
}

\section{Storm advancement coefficient determination according to origin and hazard rainfall in the Cuban archipelago}

Yoel Martínez-González1, ORCID: https://www.orcid.org/0000-00028023-7897

Eduardo O. Planos-Gutiérrez², ORCID: https://www.orcid.org/00000002-1794-7327

${ }^{1}$ Instituto Superior de Tecnología y Ciencias Aplicadas, Universidad de La Habana, La Habana, Cuba, ymg@instec.cu

${ }^{2}$ Centro del Clima, Instituto de Meteorología, La Habana, Cuba, eduardo.planos@insmet.cu

Autor para correspondencia: Yoel Martínez-González, ymg@instec.cu

\section{Resumen}


En este trabajo se presenta una generalización de la metodología de Martínez, Planos y Perdigón (2020), y se determina el coeficiente de avance de las tormentas y su relación con el hietograma de precipitación de tormentas de tipo específico. El coeficiente de avance de la tormenta es un parámetro hidrológico utilizado pormuchos autores para determinar el instante donde tiene lugar la intensidad máxima de la precipitación. Esto facilita la selección del modelo de distribución temporal de precipitaciones, criterio de gran utilidad para el diseño de obras de ingeniería y la predicción hidrológica. En este artículo se aborda el tema definiendo el coeficiente de avance según el origen y peligrosidad de la precipitación, fundamentalmente para las precipitaciones causadas por los eventos meteorológicos propios de la interacción trópico-latitudes medias, tropicales no ciclónicos y ciclónicos. Entre los resultados figura la obtención de los hietogramas de precipitación para diferentes sistemas meteorológicos, así como diversos tipos de peligrosidad, lo cual le otorga carácter climático y regional. El área de estudio abarca todo el territorio cubano.

Palabras clave: precipitaciones, coeficiente de avance de la tormenta, peligro, curvas IDF.

\section{Abstract}

In this paper, a generalization methodology of Martínez, Planos and Perdigón (2020) is presented. The storm advancement coefficient and its relationship with the specific type of storm precipitation hyetograph is determined. The storm advancement coefficient is a hydrological 
parameter used to determine the instant where the maximum rainfall intensity occurs for specific storms. This issue the selection of the rainfall temporal distribution model, which is very useful for hydraulic engineering design and hydrological prediction. The proposed approach, define the coefficient according to rainfall origin and its hazard; mainly for the meteorological events typical of the tropic interaction-middle latitudes, non-cyclonic tropical, and cyclonic. Finally, rainfall hyetographs for different hydrometeorological systems were obtained, as well as several types of hazards, which gives it a climatic and regional character. This paper is for the entire Cuban territory.

Keywords: Rainfalls, storm advancement coefficient, hazard, IDF curves.

Recibido: $27 / 01 / 2020$

Aceptado: 03/09/2020

\section{Introducción}

Los patrones conductuales de las grandes precipitaciones y las precipitaciones intensas en el archipiélago cubano han sido estudiados por Planos, Limia, Vega, Boudet y Hernández (2011), y Planos (2013), con un 
enfoque hidrometeorológico e hidrológico, basado en el origen de los eventos pluviales. Para este trabajo se asume la agrupación general de Ios sistemas meteorológicos utilizada por Planos en cuanto a: a) sistemas de interacción trópico-latitudes medias-SITLM, y b) sistemas de tipo tropical (no ciclónicos-STNC y ciclónicos-STC).

Bajo la denominación de SITLM se agrupan las situaciones sinópticas que fundamentalmente se desarrollan en el periodo poco Iluvioso (noviembre-abril), como baja extratropical, débil gradiente barométrico, zonas frontales y anticiclón típico continental. En sentido general, se puede afirmar que las precipitaciones de este origen no suelen producir con frecuencia fenómenos hidrometeorológicos relevantes, e incluso en el caso en que éstas sean significativas, la humedad antecedente en el suelo no favorece la generación de crecidas y, mucho menos, la simultaneidad de ellas en una región.

De forma análoga se definen STNC y STC para situaciones típicas de la temporada lluviosa, aunque la actividad ciclónica y de otros tipos de fenómenos meteorológicos tropicales se extiende hasta mediados de noviembre, mes comprendido entre los poco lluviosos. Los sistemas que prevalecen en esta época del año son ondas tropicales y ciclones (en este último se incluyen las depresiones tropicales), fenómenos que de manera frecuente producen copiosas e intensas precipitaciones (INSMET, 2019). Esta división (STNC y STC) fue propuesta por Planos et al. (2011) teniendo en cuenta las diferencias en la potencialidad pluvial de estos fenómenos, en términos de magnitud e intensidad, así como de la extensión del área que afectan. 
Otro enfoque relacionado con los patrones de las precipitaciones es el análisis del peligro asociado con ellas tanto en magnitud como en intensidad, por los desastres que provocan en prácticamente todos los ecosistemas naturales y humanos (Planos et al., 2011; Planos, 2013). Además de las inundaciones, existen otras consecuencias, como la pérdida de suelo por la erosión, el incremento de la sedimentación en los embalses, y la influencia en la disponibilidad de agua y en las políticas de manejo de este recurso.

Planos, Limia y Vega (2005), y Planos et al. (2011) elaboraron un mapa de peligro (Figura 1) basado en los indicadores siguientes: lámina máxima absoluta $(\mathrm{mm})$; frecuencia de láminas máximas superiores a 100 mm; lámina con periodo de retorno de 100 años $(\mathrm{mm})$, y probabilidad de una lámina de lluvia $\geq 100 \mathrm{~mm}$ en dos años. En este mapa se regionaliza el comportamiento de las precipitaciones en tres zonas de peligro (altoextremo, significativo y moderado), incluyendo un conjunto de relaciones típicas que caracterizan el comportamiento de la precipitación y su intensidad, y tablas que caracterizan las zonas determinadas. El mapa y el material complementario que le acompaña tienen un elevado valor práctico, ya que permite utilizarlo con propósitos medioambientales e ingenieriles, con particular uso en cualquiera de las aplicaciones donde el impacto de las grandes precipitaciones y las precipitaciones intensas sea nocivo, así como criterio de analogía para trabajar en zonas donde no existan datos. 
Tecnología y

Ciencias $₫$ Agua
2021, Instituto Mexicano de Tecnología del Agua

Open Access bajo la licencia CC BY-NC-SA 4.0

(https://creativecommons.org/licenses/by-nc-sa/4.0/)

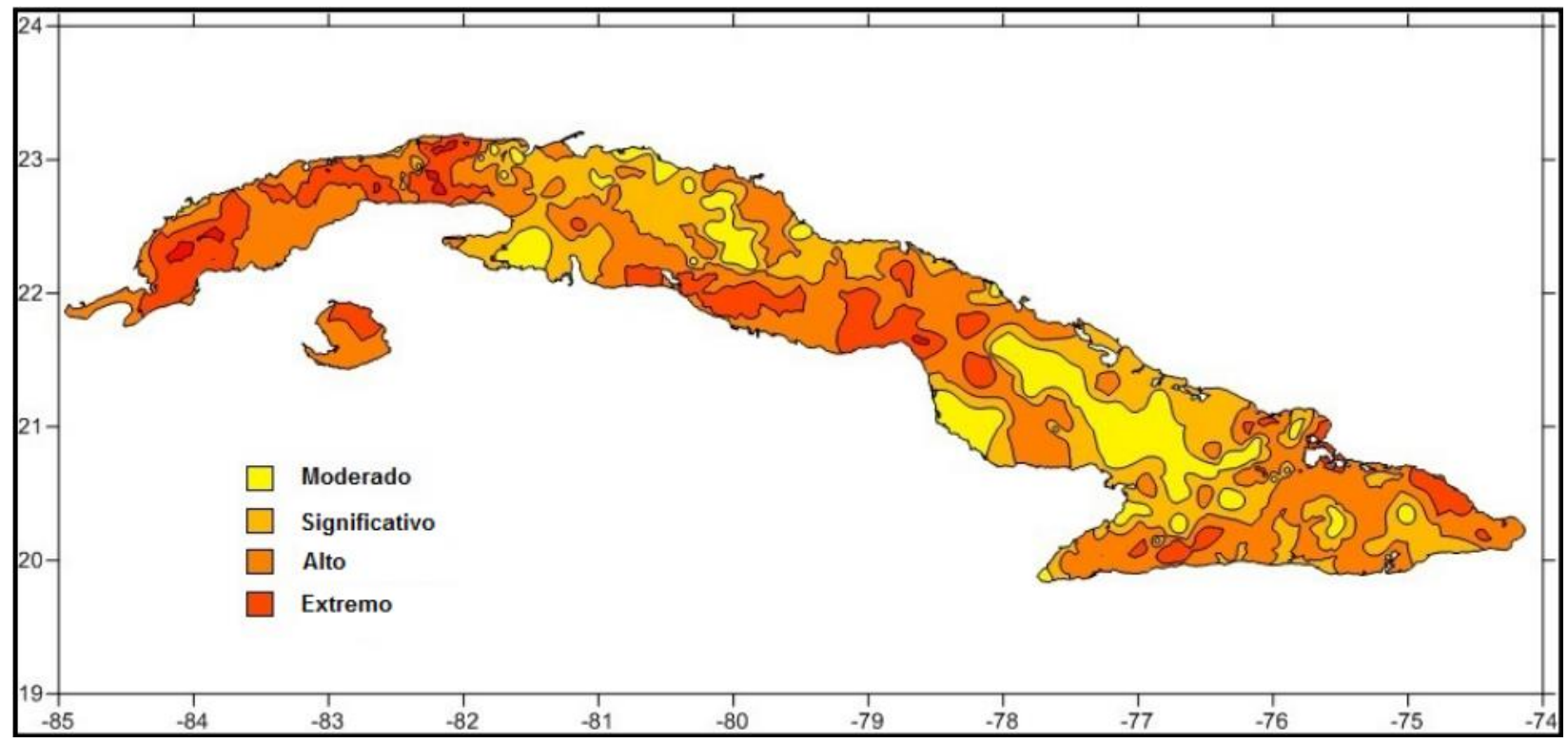

Figura 1. Mapa de peligro de precipitaciones intensas y gran magnitud. Fuente: Planos et al. (2011), Planos (2013).

Los patrones conductuales de las precipitaciones, ya sea clasificados por su origen o por el peligro asociado con su magnitud e intensidad, pueden ser representados por las curvas IDF (Martínez et al., 2020). Estas curvas, entre múltiples aplicaciones, facilitan que los diseños ingenieriles de obras civiles, y en particular las hidráulicas, sean más confiables (Gutiérrez, Pérez, Angulo, Chiriboga, \& Valdés, 2015; Balbastre, 2018), y constituye una herramienta de análisis y planificación en el corto, mediano y largo plazos, que tiene un peso significativo en las decisiones de las inversiones y su protección. En la Tabla 1 se muestra un grupo genérico de ecuaciones utilizadas en la práctica hidrológica para estimar la intensidad de la precipitación (Minh-Nhat, Tachikawa, \& Takara, 2006). Según estas ecuaciones, la intensidad de la precipitación decrece con la 
Tecnología y

Ciencias $\stackrel{\unlhd}{\unlhd}$ gua
2021, Instituto Mexicano de Tecnología del Agua

Open Access bajo la licencia CC BY-NC-SA 4.0

(https://creativecommons.org/licenses/by-nc-sa/4.0/)

duración del evento lluvioso, lo cual se puede asociar con cierta probabilidad de ocurrencia.

Tabla 1. Ecuaciones empíricas para curvas IDF. Fuente: Martínez et al. (2020).

\begin{tabular}{|c|c|}
\hline Autor & Formulación \\
\hline Sherman (1931) & $I=\frac{a}{(t+b)^{n}}(1 \mathrm{a})$ \\
\hline Bernard (1932) & $I=\frac{a}{t^{n}} \quad(1 \mathrm{~b})$ \\
\hline Linsley, Kohler y Paulhus (1949) & $I=\frac{a}{t+b} \quad(1 \mathrm{c})$ \\
\hline Wenzel (1982) & $I=\frac{a}{t^{n}+b} \quad(1 \mathrm{~d})$ \\
\hline $\begin{array}{c}a, b \text { y } n=\text { parámetros de ajuste relacionados con los datos, en sentido } \\
\text { general varían con la probabilidad de ocurrencia }\end{array}$ \\
\hline
\end{tabular}

No obstante la contribución de Planos et al. (2011), Planos (2013) y el reciente trabajo de Martínez et al. (2020) relacionado con STC, todavía persiste un vacío en el estudio del comportamiento de otros tipos de sistemas hidrometeorológicos (SITLM y STNC), y el peligro asociado con éstos. Planos et al. (2011) hacen una detallada caracterización de las grandes precipitaciones y las precipitaciones intensas, según su origen; mientras que Martínez et al. (2020) emplean el modelo de Wenzel para obtener los parámetros de las curvas IDF para los STC, construyen los hietogramas adimensionales de precipitación y estiman el coeficiente de avance para este tipo de sistemas. 
Teniendo en cuenta lo anterior, el objetivo de este trabajo es determinar el instante de tiempo donde tiene lugar la intensidad máxima de la precipitación -caracterizada por el coeficiente de avance de la tormenta en los sistemas meteorológicos responsables de situaciones de peligro en Cuba- y obtener los hietogramas de precipitación correspondientes.

\section{Materiales y métodos}

Al realizar una inspección en las ecuaciones (1a) a (1d) es posible extender la estructura de las mismas, considerando que cada parámetro de ajuste varía con la probabilidad de ocurrencia, utilizando el siguiente modelo:

$I=\frac{a}{\left(t^{n}+b\right)^{c}}$

En la Tabla 2 se muestran los valores que deben adoptar los parámetros de la Ecuación (2), para describir los modelos de curvas IDF de Linsley, Kohler y Paulhus (1949), Bernard (1932), Sherman (1931) y Wenzel (1982), presentados con anterioridad en la Tabla 1. 
Tabla 2. Valores de los parámetros de la Ecuación (2) para casos particulares.

\begin{tabular}{|c|c|}
\hline Autor & Parámetros \\
\hline Sherman (1931) & $n=1$ \\
& $c:$ reemplaza a $n$ en (1a) \\
\hline Bernard (1932) & $b=0, c=1$ \\
\hline Linsley, Kohler y Paulhus & $n=1, c=1$ \\
\hline Wenzel (1989) & $c=1$ \\
\hline
\end{tabular}

El empleo de curvas adimensionales es un recurso técnico de excelencia para lo siguiente: hacer comparables los registros en localidades físico-geográficamente homogéneas; dar mayor certidumbre a otras técnicas, como la transposición de valores, y tratar los valores anómalos (Martínez et al., 2020). Para hacer esto, primero se normalizan los tiempos e intensidades. En este caso, se considera la duración $T_{d}=1$ 440 min y la intensidad media $I_{m}$ para cada probabilidad de ocurrencia como $I_{m}($ prob $)=\frac{P_{a c u m}(\text { prob })}{T_{d}}$, siendo $P_{\text {acum }}$ la precipitación acumulada en $T_{d}$. De esta manera se establecen las siguientes relaciones adimensionales $\tau=\frac{t}{T_{d}}$ e $i=\frac{I}{I_{m}}$, para tiempo e intensidad, respectivamente, y entonces la Ecuación (2) se puede reescribir como: 
Tecnología y

Ciencias $\stackrel{\unlhd}{\Im}$ Agua

$i=\frac{a}{\left(\tau^{n}+b\right)^{c}}$
2021, Instituto Mexicano de Tecnología del Agua

Open Access bajo la licencia CC BY-NC-SA 4.0

(https://creativecommons.org/licenses/by-nc-sa/4.0/)

Planos et al. (2011) demuestran que existe un patrón identificable en las relaciones temporales y espaciales de las precipitaciones en el archipiélago cubano, lo que permitió a dichos autores determinar relaciones tipo en localidades homogéneas. Dentro de éstas se presentan relaciones tipos que son de extendido uso ingenieril, teniendo especial relevancia las curvas IDF, así como el coeficiente de avance de la tormenta $r$. Las curvas IDF se obtienen a partir de las características de las precipitaciones según su origen, peligro y probabilidad de ocurrencia. El conocimiento de las precipitaciones asociadas con diversos sistemas hidrometeorológicos y tipos de peligro puede establecer criterios de diseño para diversas obras de ingeniería.

En esta contribución, de forma análoga a lo desarrollado por Martínez et al. (2020), se determinaron los parámetros de las curvas IDF, correspondientes al modelo de Wenzel (Ecuación (1d)), sin restarle generalidad al procedimiento aquí presentado, el cual se sustenta sobre el modelo general de curvas IDF dado por la Ecuación (3). En la Tabla 3 y Tabla 4 se muestran los valores de $a, b$ y $n$ a partir de los resultados de Planos et al. (2011), según el origen y peligro de las precipitaciones, caracterizados por las altas correlaciones obtenidas al aplicar el método de Levenberg-Marquardt, descrito en detalle por Gill, Murray y Wright (1981) para minimizar la suma de los errores cuadráticos. 
Tecnología y

Ciencias $₫$ Agua
2021, Instituto Mexicano de Tecnología del Agua

Open Access bajo la licencia CC BY-NC-SA 4.0

(https://creativecommons.org/licenses/by-nc-sa/4.0/)

Tabla 3. Parámetros de curvas IDF del modelo de Wenzel según el origen y probabilidad de ocurrencia de las precipitaciones.

\begin{tabular}{|c|c|c|c|c|c|}
\hline \multirow{2}{*}{ Parámetros } & \multicolumn{5}{|c|}{ Sistemas de interacción trópico- latitudes medias (SITLM) } \\
\cline { 2 - 6 } & prob = 0.999 & prob = 0.99 & prob = 0.94 & prob = 0.8 & prob = 0.5 \\
\hline$a$ & 1.0055459 & 1.00318185 & 0.99964234 & 0.9956794 & 0.99411433 \\
\hline$b$ & 0.04493726 & 0.04177178 & 0.03626707 & 0.031846 & 0.02408982 \\
\hline$n$ & 0.8202653 & 0.82030316 & 0.82711352 & 0.81811769 & 0.83950346 \\
\hline$R^{2}$ & 0.99601815 & 0.99630588 & 0.99689359 & 0.99689332 & 0.99735852 \\
\hline \multirow{2}{*}{ Parámetros } & \multicolumn{5}{|c|}{ Sistemas tropicales no ciclónicos (STNC) } \\
\cline { 2 - 6 } & prob $=\mathbf{0 . 9 9 9}$ & prob = 0.99 & prob = 0.94 & prob = 0.8 & prob = 0.5 \\
\hline$a$ & 1.0255523 & 1.0264221 & 1.0259757 & 1.02858103 & 1.03104293 \\
\hline$b$ & 0.0587669 & 0.0573078 & 0.05519624 & 0.05247556 & 0.0451634 \\
\hline$n$ & 0.7820167 & 0.7827663 & 0.78093098 & 0.78114111 & 0.81388196 \\
\hline$R^{2}$ & 0.998627 & 0.9996138 & 0.99976852 & 0.99985955 & 0.99999486 \\
\hline \multirow{2}{*}{ Parámetros } & & \multicolumn{5}{|c|}{ Sistemas tropicales ciclónicos (STC) } \\
\cline { 2 - 6 } & prob $=\mathbf{0 . 9 9 9}$ & prob = 0.99 & prob = 0.94 & prob = 0.8 & prob = 0.5 \\
\hline$R^{2}$ & 1.081156 & 1.0691529 & 1.07666973 & 1.04339008 & 0.99610462 \\
\hline$b$ & 0.070814 & 0.0688126 & 0.05684803 & 0.05877438 & 0.05241834 \\
\hline$n$ & 0.651096 & 0.6037224 & 0.74003492 & 0.59413043 & 0.72812478 \\
\hline & 0.997321 & 0.9979585 & 0.99833002 & 0.99837534 & 0.99867582 \\
\hline
\end{tabular}

Tabla 4. Parámetros de curvas IDF del modelo de Wenzel según el peligro y probabilidad de ocurrencia de las precipitaciones. 
Tecnología y

Ciencias $\stackrel{\unlhd}{\unlhd}$ gua
2021, Instituto Mexicano de Tecnología del Agua

Open Access bajo la licencia CC BY-NC-SA 4.0

(https://creativecommons.org/licenses/by-nc-sa/4.0/)

\begin{tabular}{|c|c|c|c|c|}
\hline \multirow{2}{*}{ Parámetros } & \multicolumn{4}{|c|}{ Peligro moderado } \\
\hline & prob $=0.2$ & prob $=0.1$ & prob $=0.02$ & prob $=0.01$ \\
\hline$a$ & 1.01836241 & 1.00646802 & 1.01176135 & 1.0161458 \\
\hline$b$ & 0.01716559 & 0.02224011 & 0.02633496 & 0.02652005 \\
\hline$n$ & 1.02622485 & 0.96645452 & 0.95334331 & 0.9875706 \\
\hline$R^{2}$ & 0.99979561 & 0.99985808 & 0.99980559 & 0.99990775 \\
\hline \multirow{2}{*}{ Parámetros } & \multicolumn{4}{|c|}{ Peligro significativo } \\
\hline & prob $=0.2$ & prob $=0.1$ & prob $=0.02$ & prob $=0.01$ \\
\hline$a$ & 1.00558929 & 1.00974856 & 1.03131025 & 1.04442169 \\
\hline$b$ & 0.01787631 & 0.02183508 & 0.02725466 & 0.03495176 \\
\hline$n$ & 1.07872786 & 0.95976548 & 0.97317074 & 0.88854247 \\
\hline$R^{2}$ & 0.99856711 & 0.99996518 & 0.99942973 & 0.99863044 \\
\hline \multirow{2}{*}{ Parámetros } & \multicolumn{4}{|c|}{ Peligros alto y extremo } \\
\hline & prob $=0.2$ & prob $=0.1$ & prob $=0.02$ & prob $=0.01$ \\
\hline$a$ & 1.00971041 & 1.00974856 & 1.03131025 & 1.04442169 \\
\hline$b$ & 0.01557916 & 0.02183508 & 0.02725466 & 0.03495176 \\
\hline$n$ & 0.97415468 & 0.95976548 & 0.97317074 & 0.88854247 \\
\hline$\overline{R^{2}}$ & 0.99890429 & 0.99996518 & 0.99942973 & 0.99863044 \\
\hline
\end{tabular}

\section{Formulación general para la determinación del coeficiente de avance de la tormenta}


Keifer y Chu (1957) desarrollaron un modelo de intensidad instantánea basado en las curvas IDF, descritas en detalle por Chow, Maidment y Mays (1994). Este modelo fue aplicado por Na y Yoo (2018) para evaluar la distribución temporal de precipitaciones con eventos máximos anuales en Seúl, Corea, así como por Martínez et al. (2020) para los STC. Al aplicar este modelo a la Ecuación (3) puede demostrarse que la intensidad instantánea $i$ vendrá dada, de forma general, por:

$i=\frac{a\left[b+(1-c n) \zeta^{n}\right]}{\left(b+\zeta^{n}\right)^{c+1}}$

En la expresión anterior se introduce la variable $\zeta$, la cual representa una escala temporal relativa al coeficiente de avance de la tormenta $r, y$ que se define como:

$\zeta= \begin{cases}\frac{r-\tau}{r} & 0 \leq \tau \leq r \\ \frac{\tau-r}{1-r} & r \leq \tau \leq 1\end{cases}$

El coeficiente de avance de la tormenta, $r$, se define como la proporción del tiempo al pico de la tormenta respecto a su duración total. Este coeficiente precisa una asimetría en la forma de la tormenta en el intervalo $0<r<1$. De igual forma, $r$ delimita el punto en el que la 
tormenta con duración $T_{d}$ alcanza la máxima intensidad de Iluvia (Chow et al., 1994).

El procedimiento tradicional para determinar el parámetro $r$ es calcular la relación entre el tiempo de intensidad máxima y la duración total de la tormenta para una serie de tormentas $(j=1,2, \ldots m)$ de diferentes duraciones $T_{d j}$. La media de estas relaciones, ponderada de acuerdo con la duración de cada evento, es el valor representativo de $r$ (Chow et al., 1994):

$\bar{r}=\frac{\sum_{j=1}^{m} r_{j} T_{d j}}{\sum_{j=1}^{m} T_{d j}}$

Wenzel (1982), citado además por Chow et al. (1994), reporta valores del coeficiente de avance de la tormenta para varios lugares de EUA y la India. Watt, Chow, Hogg y Lathem (1986), citados por Balbastre (2018), sugieren para distintas ubicaciones en Canadá valores entre 0.22 y 0.52. Guevara y Carballo (2008) reportan para Venezuela un valor de $r=0.41$, con una desviación estándar de 0.075 .

En Cuba se registran escasas investigaciones en la determinación de $r$. Cruz (2001) reporta un valor de $r=0.327$ tras analizar 1543 tormentas que afectaron a los territorios de Mayabeque, Artemisa y La Habana. Planos et al. (2011), a partir de registros de 10 tormentas de origen ciclónico que afectaron al archipiélago cubano, determinaron $r=$ 
0.77, evidenciando que la intensidad máxima de dichos sistemas hidrometeorológicos ocurre en el cuartil horario final de los mismos.

Martínez et al. (2020) proponen un procedimiento analítico para estimar dicho parámetro, partiendo del hietograma obtenido por el método de la intensidad instantánea. De forma análoga, dicho procedimiento será extendido y generalizado a los sistemas abordados en esta contribución, relacionado además con el peligro de las precipitaciones, utilizando el modelo general de intensidad, definido por la Ecuación (4). La intensidad máxima se obtiene evaluando para $\tau=r$ (equivalente $\mathrm{a} \zeta=0$ ), o sea:

$i(0)=\frac{a}{b^{c}}$

El hietograma descrito por la Ecuación (4) es una función por tramos, es continua en $\zeta=0$, pero no es derivable, ya que $\left.\frac{d i}{d \zeta}\right|_{\zeta=0}=\infty$. Por esta razón, Martínez et al. (2020) proponen establecer una vecindad en torno a $\zeta=0$, es decir, $\zeta=\frac{\varepsilon}{r}$ y para el modelo en discusión resulta:

$i\left(\frac{\varepsilon}{r}\right)=k \frac{a}{b^{c}}$

siendo $k$ un coeficiente muy próximo a 1, por lo que la Ecuación (4) puede rescribirse como: 
$k \frac{a}{b^{c}}=\frac{a\left[b+(1-c n)\left(\frac{\varepsilon}{r}\right)^{n}\right]}{\left[b+\left(\frac{\varepsilon}{r}\right)^{n}\right]^{c+1}}$

Para $a, b, c, n$ conocidos se impone un valor de $k$ suficientemente próximo a la unidad (p. ej., $k=0.95 \div 0.99$ ) y la Ecuación (9) puede resolverse por algún método numérico de separación de raíces conocido, en términos de la variable $\varepsilon / r$. La dependencia de $a, b, c, n$ con la probabilidad de ocurrencia de las precipitaciones según su origen condiciona a la vecindad $\varepsilon / r$ funcionalmente dependiente de la probabilidad de ocurrencia de dicha variable hidrometeorológica. Para el caso particular de $c=1$ (modelo de curvas IDF propuesto por Wenzel), se puede demostrar fácilmente que la Ecuación (9) se reduce a la forma:

$(1-k) b^{2}+(1-n-2 k) b\left(\frac{\varepsilon}{r}\right)^{n}-k\left(\frac{\varepsilon}{r}\right)^{2 n}=0$

En la Ecuación (10) se introduce la sustitución $z=\left(\frac{\varepsilon}{r}\right)^{n}$ y ésta queda reducida a un polinomio de segundo grado, cuyas raíces $z_{1}$ y $z_{2}$ quedan determinadas por:

$z_{1,2}=\left\{\frac{b}{2 k}\left[2 k-(1-n) \pm \sqrt{(1-n-2 k)^{2}+4 k(1-k)}\right]\right\}^{1 / n}$ 
Martínez et al. (2020) destacan que el valor de $r$ tiene carácter regional y, a la vez, probabilístico, pues depende de parámetros asociados con las curvas IDF. También está determinado por los niveles de aproximación prestablecidos por los coeficientes $\varepsilon$ y $k$. Esto dificulta la determinación de $r$ a través de las ecuaciones (9) u (11), porque los parámetros $\varepsilon$ y $k$ están correlacionados. Por tal razón, en la vecindad de $r$ se diferencia la Ecuación (4) respecto a $\tau$ y se evalúa para $\tau=r-\varepsilon$, es decir:

$$
\left.\frac{d i}{d \tau}\right|_{\tau=r-\varepsilon}=\frac{(c+1) a n\left[b-(c n-1)\left(\frac{\varepsilon}{r}\right)^{n}\right]\left(\frac{\varepsilon}{r}\right)^{n-1}}{r\left[b+\left(\frac{\varepsilon}{r}\right)^{n}\right]^{c+2}}+\frac{a n(c n-1)\left(\frac{\varepsilon}{r}\right)^{n-1}}{r\left[b+\left(\frac{\varepsilon}{r}\right)^{n}\right]^{c+1}}
$$

En la vecindad considerada, la Ecuación (12) puede aproximarse por:

$\left.\frac{d i}{d \tau}\right|_{\tau=r-\varepsilon} \cong \frac{(1-k) \frac{a}{b^{c}}}{\frac{\varepsilon}{r}}$

Igualando las ecuaciones (12) y (13) es posible obtener la ecuación general para el coeficiente de avance de la tormenta, aplicable a cualquier origen y peligro de las precipitaciones:

$r=\frac{b^{c}}{(1-k)}\left(\frac{\varepsilon}{r}\right)\left\{\frac{(c+1) n\left[b-(c n-1)\left(\frac{\varepsilon}{r}\right)^{n}\right]\left(\frac{\varepsilon}{r}\right)^{n-1}}{\left[b+\left(\frac{\varepsilon}{r}\right)^{n}\right]^{c+2}}+\frac{n(c n-1)\left(\frac{\varepsilon}{r}\right)^{n-1}}{\left[b+\left(\frac{\varepsilon}{r}\right)^{n}\right]^{c+1}}\right\}$ 
Siendo $\varepsilon / r$ solución numérica de la Ecuación (9), tal que $\varepsilon / r>0$. Para $c=1$, la Ecuación (14) se reduce a la obtenida por Martínez et al. (2020) para determinar el coeficiente de avance de la tormenta $r$, esto es:

$r=\frac{b}{(1-k)}\left(\frac{\varepsilon}{r}\right)\left\{\frac{2 n\left[b-(n-1)\left(\frac{\varepsilon}{r}\right)^{n}\right]\left(\frac{\varepsilon}{r}\right)^{n-1}}{\left.\left[b+\frac{\varepsilon}{r}\right)^{n}\right]^{3}}+\frac{n(n-1)\left(\frac{\varepsilon}{r}\right)^{n-1}}{\left[b+\left(\frac{\varepsilon}{r}\right)^{n}\right]^{2}}\right\}$

En este caso, $\varepsilon / r$ es la raíz positiva de la Ecuación (11), es decir:

$\frac{\varepsilon}{r}=\left\{\frac{b}{2 k}\left[2 k-(1-n)+\sqrt{(1-n-2 k)^{2}+4 k(1-k)}\right]\right\}^{1 / n}$

En las ecuaciones (14) y (15), $r=r(k$, prob), pues los parámetros $b$, c y $n$ varían con la probabilidad de ocurrencia, según sea el origen de las precipitaciones. En la Figura 2 se muestra gráficamente la interpretación geométrica del procedimiento propuesto, considerando $\frac{I}{I_{m}}=$ $f(\zeta)$. 


\section{1, Instituto Mexicano de Tecnología del Agua}

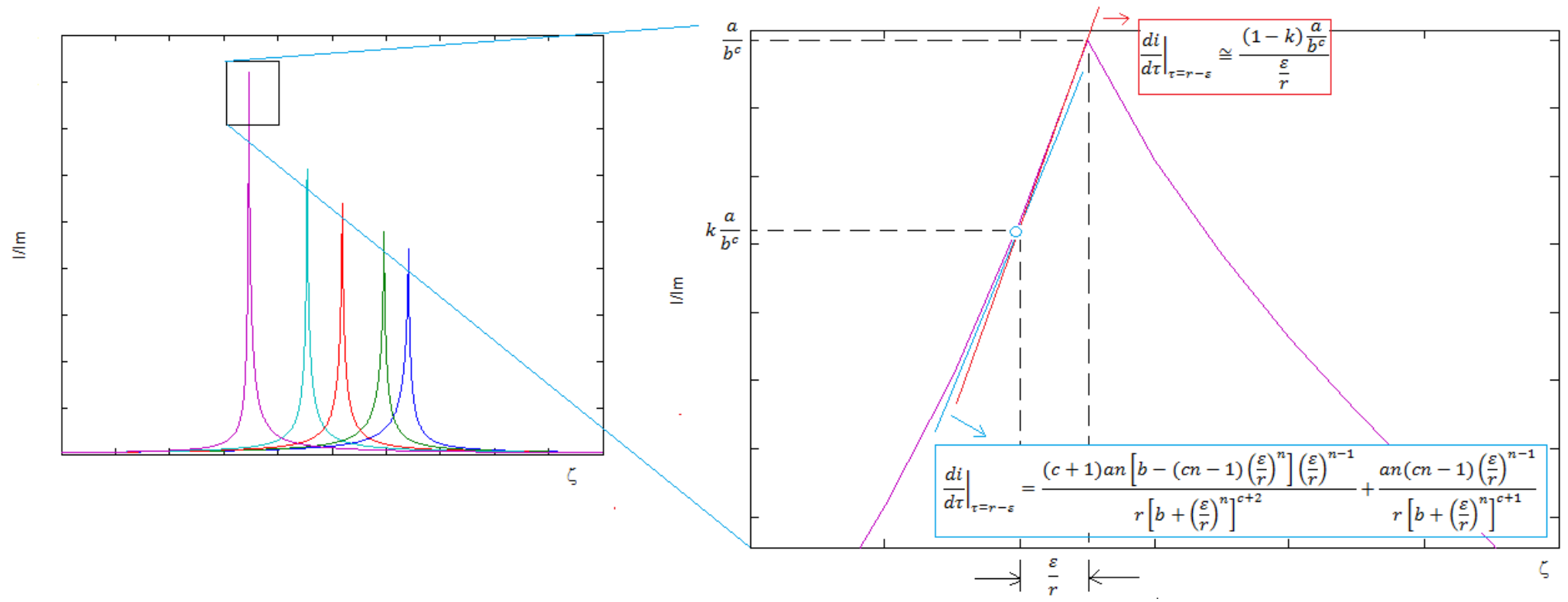

Figura 2. Interpretación geométrica del procedimiento propuesto.

\section{Resultados y discusión}

Una vez conocidos los parámetros $a, b, n$ para determinar el coeficiente de avance de la tormenta $r$ (Tabla 5 ) pueden aplicarse las ecuaciones (14) o (15), haciendo $k=0.99$. En este caso, para ser consecuente al proceso de estimación de parámetros del modelo de curvas IDF de Wenzel, se aplicará la Ecuación (15), y de acuerdo con el origen y peligro de las precipitaciones se obtienen los resultados que se muestran en la Figura 3, Figura 4, Figura 5 y Figura 6. 
Tabla 5. Valores de $r$ según el origen y probabilidad de las precipitaciones.

\begin{tabular}{|c|c|c|c|}
\hline \multirow{2}{*}{ Prob. } & \multicolumn{3}{|c|}{ Coeficiente de avance de la tormenta $\boldsymbol{r}$} \\
\cline { 2 - 4 } & SITLM & STNC & STC \\
\hline 0.999 & 0.6413 & 0.8243 & 0.9091 \\
\hline 0.99 & 0.5961 & 0.8042 & 0.8445 \\
\hline 0.94 & 0.5190 & 0.7738 & 0.7792 \\
\hline 0.8 & 0.4541 & 0.7358 & 0.7141 \\
\hline 0.5 & 0.3463 & 0.6428 & 0.7131 \\
\hline
\end{tabular}


2021, Instituto Mexicano de Tecnología del Agua

Open Access bajo la licencia CCBY-NC-SA 4.0

(https://creativecommons.org/licenses/by-nc-sa/4.0/)

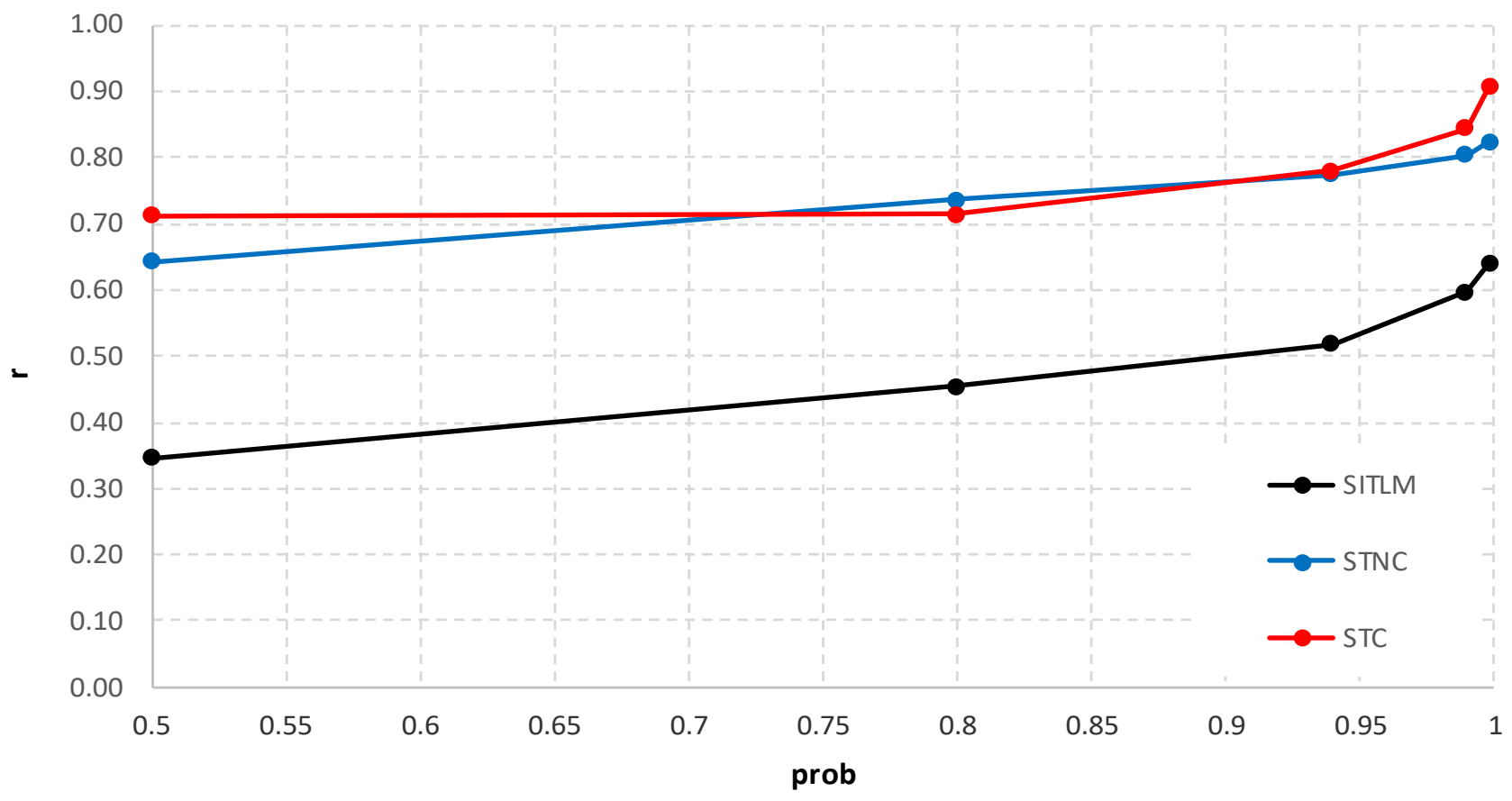

Figura 3. Tendencia del coeficiente de avance de la tormenta según el origen y la probabilidad de ocurrencia de las precipitaciones. 
Tecnología y

Ciencias $₫$ Agua
2021, Instituto Mexicano de Tecnología del Agua

Open Access bajo la licencia CCBY-NC-SA 4.0

(https://creativecommons.org/licenses/by-nc-sa/4.0/)
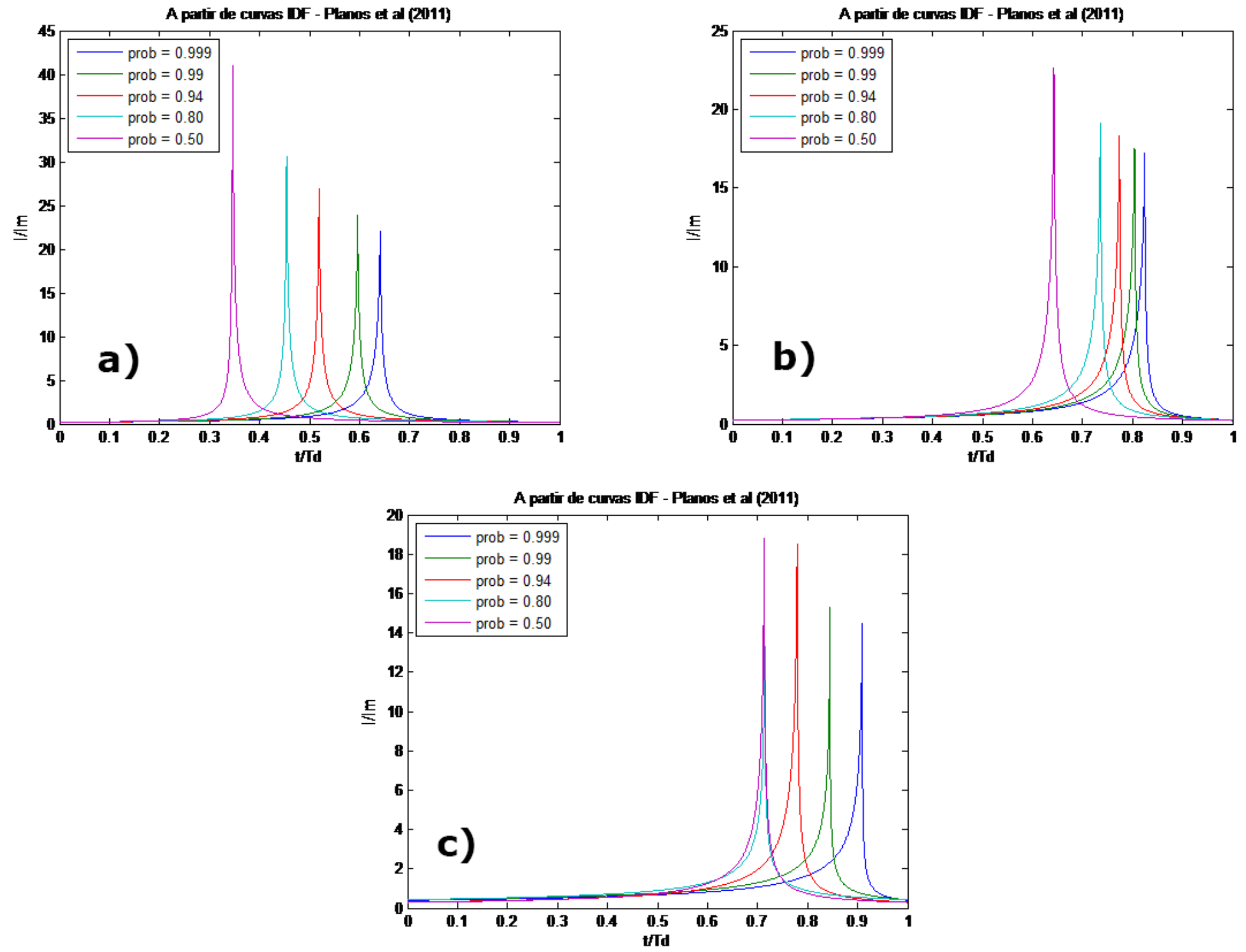

Figura 4. Hietogramas adimensionales para las probabilidades de ocurrencia de: a) SITLM; b) STNC; c) STC. 
2021, Instituto Mexicano de Tecnología del Agua

Open Access bajo la licencia CC BY-NC-SA 4.0

(https://creativecommons.org/licenses/by-nc-sa/4.0/)

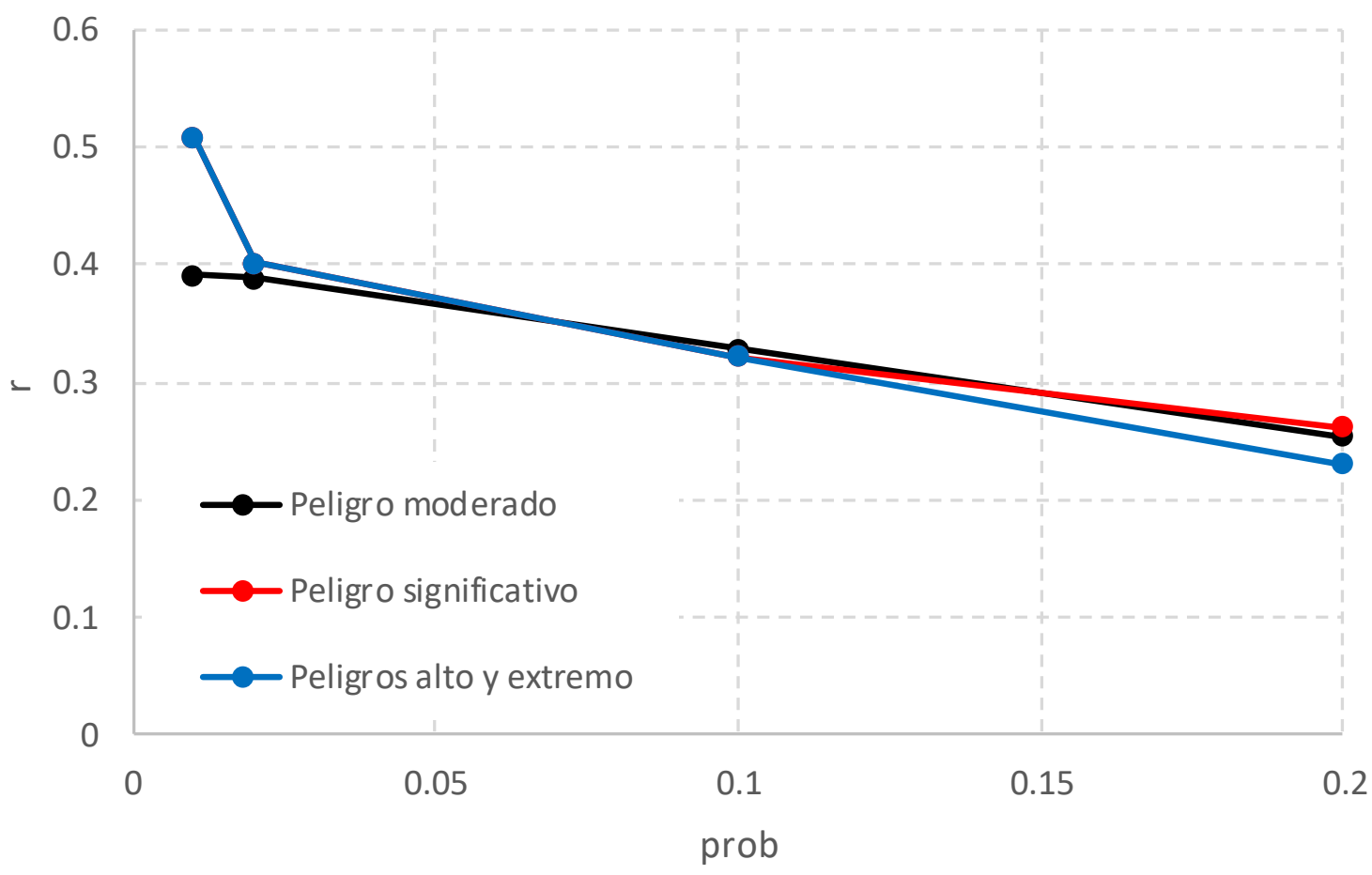

Figura 5. Tendencia del coeficiente de avance de la tormenta según el peligro y probabilidad de ocurrencia de las precipitaciones. 
Tecnología y

Ciencias $\stackrel{\varpi}{\triangleleft}$ Agua
2021, Instituto Mexicano de Tecnología del Agua

Open Access bajo la licencia CCBY-NC-SA 4.0

(https://creativecommons.org/licenses/by-nc-sa/4.0/)
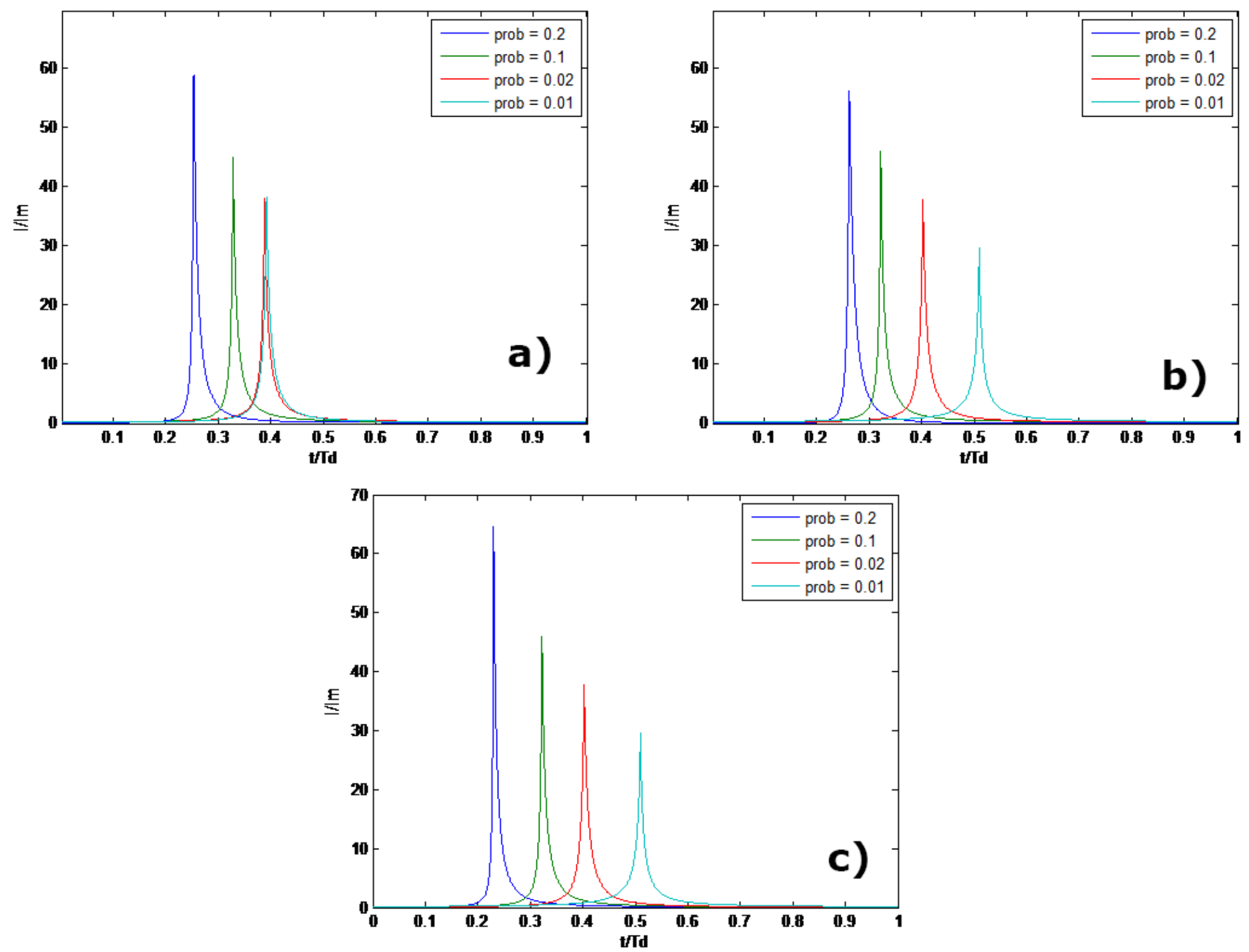

Figura 6. Hietogramas adimensionales para las probabilidades de ocurrencia asociadas con precipitaciones de: a) peligro moderado; b) peligro significativo; c) peligros alto y extremo. 
Como se aprecia en las Figura 4 (a-c), de acuerdo con Chow et al. (1994), para prob < 0.94, los SITLM tienden a ser de tipo avanzado, ya que $r<0.5$, lo cual significa que la intensidad máxima ocurre antes del punto medio de duración total de la tormenta. En el resto de las probabilidades y tipos de sistemas (STNC y STC), debido a que $r>0.5$, la intensidad máxima ocurre después de la mitad de duración de la tormenta y por tanto los sistemas son de tipo retardado (Figura 4). Otro aspecto de interés es que en el rango de probabilidades analizado el coeficiente de avance de la tormenta para los sistemas tropicales ciclónicos y no ciclónicos es muy similar. Además, en términos adimensionales, las intensidades máximas son del mismo orden. Estos sistemas, como ya se explicó, se diferencian fundamentalmente en su potencial pluvial, así como la extensión del área que afectan.

El enfoque relacionado con la peligrosidad de las precipitaciones aporta nuevos elementos, pues considera el efecto regional de áreas de igual peligro. El patrón conductual de precipitaciones intensas y de gran magnitud, en sentido general, arroja hietogramas de tipo avanzado para los distintos tipos de peligro establecidos (moderado, significativo, alto y extremo), en el rango de probabilidades de 0.01 a 0.2. Es válido destacar que, con el incremento de la peligrosidad y la disminución de la probabilidad, existe una tendencia a la simetría en los hietogramas. Los valores comprendidos entre 0.01 a 0.2 reflejan la probabilidad de sobrepasar cierto valor de precipitación (acorde con Planos et al., 2011, $100 \mathrm{~mm}$ ), ya que marca la tendencia del coeficiente de avance de la tormenta en el rango analizado (Figura 5). 
Los valores de $r$ obtenidos (Tabla 6), así como los hietogramas adimensionales de la Figura 6 (a-c) pueden ser aplicados a las áreas de peligro del mapa de la Figura 1, según sea el caso, lo cual permitirá establecer estudios de inundaciones en cuencas hidrográficas del archipiélago cubano.

Tabla 6. Valores de $r$ según el peligro y la probabilidad de las precipitaciones.

\begin{tabular}{|c|c|c|c|}
\hline \multirow{2}{*}{ Prob. } & \multicolumn{2}{|c|}{ Coeficiente de avance de la tormenta $r$} \\
\cline { 2 - 4 } & $\begin{array}{c}\text { Peligro } \\
\text { moderado }\end{array}$ & $\begin{array}{c}\text { Peligro } \\
\text { significativo }\end{array}$ & $\begin{array}{c}\text { Peligros } \\
\text { alto y extremo }\end{array}$ \\
\hline 0.20 & 0.2534 & 0.2623 & 0.2301 \\
\hline 0.10 & 0.3283 & 0.3222 & 0.3222 \\
\hline 0.02 & 0.3883 & 0.4025 & 0.4025 \\
\hline 0.01 & 0.3919 & 0.5098 & 0.5098 \\
\hline
\end{tabular}

\section{Conclusiones}


2021, Instituto Mexicano de Tecnología del Agua

Open Access bajo la licencia CC BY-NC-SA 4.0

(https://creativecommons.org/licenses/by-nc-sa/4.0/)

Como resultado de la aplicación del procedimiento analítico desarrollado para la determinación del coeficiente de avance de la tormenta y construir los hietogramas de precipitaciones se logró determinar el instante de tiempo donde tiene lugar la intensidad máxima de la precipitación en cada uno de los sistemas meteorológicos analizados en este trabajo, lo cual facilita la selección del modelo de distribución temporal de precipitaciones, de manera que:

- Para prob < 0.94, los SITLM tienden a ser de tipo avanzado (Chow et al., 1994), En este caso $r<0.5$, lo que significa que la intensidad máxima ocurre antes del punto medio de duración total de la tormenta.

- En el resto de las probabilidades y tipos de sistemas (STNC y STC), la intensidad máxima ocurre después de la mitad de duración de la tormenta y por tanto los sistemas son de tipo retardado debido a que $r>0.5$.

El análisis de los hietogramas de las precipitaciones intensas y de gran magnitud demuestran que:

- Son de tipo avanzado para los distintos tipos de peligro establecidos (moderado, significativo, alto y extremo), en el rango de probabilidades de 0.01 a 0.2 .

- Con el incremento de la peligrosidad y la disminución de la probabilidad existe una tendencia a la simetría en los hietogramas.

- Los valores comprendidos entre 0.01 y 0.2 reflejan la probabilidad de sobrepasar cierto valor de precipitación (acorde con Planos et al., 2011, $100 \mathrm{~mm}$ ), pues marca la tendencia del coeficiente de avance de la tormenta en el rango analizado. 
Tecnología y

Ciencias $₫$ Agua
2021, Instituto Mexicano de Tecnología del Agua

Open Access bajo la licencia CC BY-NC-SA 4.0

(https://creativecommons.org/licenses/by-nc-sa/4.0/)

\section{Referencias}

Balbastre, R. (2018). Análisis comparativo de metodologías de cálculo de tormentas de diseño para su aplicación en hidrología urbana (tesis de maestría). Universidad Politécnica de Valencia, España. Recuperado de https://riunet.upv.es/handle

Bernard, M. (1932). Formulas for rainfall intensities of long durations. Transactions of the American Society of Civil Engineers, 96, 592-624.

Chow, V. T., Maidment, D., \& Mays, L. (1994). Hidrología aplicada. Santafé de Bogotá, Colombia: McGraw-Hill Interamericana, S.A.

Cruz, M. (2001). Determinación de la tormenta de diseño. Ingeniería Hidráulica y Ambiental, 22(2), 27-30.

Gill, P.E., Murray, W., \& Wright, M. H. (1981). Practical optimization. London, UK: Academic Press.

Guevara, E., \& Carballo, N. (2008). Modelo IDF regional para el estado Cojedes, Venezuela. Revista Ingeniería UC, 15(3), 53-63, Recuperado de https://www.redalyc.org/pdf/707/70712293007.pdf

Gutiérrez, J., Pérez, F., Angulo, G., Chiriboga, G., \& Valdés, L. (2015). Determinación de las Curvas de Intensidad-Duración-Frecuencia (IDF) para la ciudad Cartagena de Indias en Colombia durante el periodo comprendido entre los años 1970 y 2015. Recuperado de https://www.researchgate.net/publication/318574137_Determinaci on_de_las_Curvas_de_Intensidad_Duracion_Frecuencia_IDF_para_I a_ciudad_Cartagena_de_Indias_en_Colombia_durante_el_periodo_ comprendido_entre_los_anos_1970_y_2015 
INSMET, Instituto de Meteorología. (2019). Generalidades de los ciclones tropicales. Recuperado de http://www.insmet.cu/asp/genesis. asp?TB0 =PLANTILLAS\&TB1 =OP TION\&TB2=/contenidos/ciclones\%20tropicales/generalidades/gener alidades.htm

Keifer, C. J., \& Chu, H. H. (1957). Synthetic storm pattern for drainage design. Journal of the Hydraulics Division, 83, 1-25.

Linsley, R., Kohler, M., \& Paulhus, J. (1949). Applied hydrology. New York, USA: McGraw-Hill.

Martínez, Y., Planos, E., \& Perdigón, D. (2020). Hietogramas adimensionales para ciclones tropicales que afectan al archipiélago cubano. Ingeniería Hidráulica y Ambiental, 41(2), Recuperado de http://scielo.sld.cu/scielo.php?script=sci_arttext\&pid=S168003382020000200048

Minh-Nhat, L., Tachikawa, Y., \& Takara, K. (2006). Establishment of intensity-duration-frequency curves for precipitation in the Monsoon Area of Vietnam. In: Annual Report of Disaster Prevention Research Institute (No. 49 B) (pp. 93-103). Kyoto, Japan: Kyoto University.

Na, W., \& Yoo, C. (2018). Evaluation of rainfall temporal distribution models with annual maximum rainfall events in Seoul, Korea. Water 10, 1468. DOI: $10.3390 / w 10101468$

Planos, E., Limia, M., \& Vega, R. (2005). Intensidad de las precipitaciones en Cuba. Informe científico del Instituto de Meteorología. La Habana, Cuba: Instituto de Meteorología. 
Planos, E., Limia, M., Vega, R., Boudet, D., \& Hernández, M. (2011). Las grandes precipitaciones y las precipitaciones intensas en Cuba: causas y características. Informe científico del Proyecto 0801. La Habana, Cuba: Instituto de Meteorología.

Planos, E. (marzo, 2013). Enfoque integrado de los estudios de peligro por grandes precipitaciones y precipitaciones intensas. Aqua-LAC, 5(1), 88-96.

Sherman, C. (1931). Frequency and intensity of excessive rainfall at Boston, Mass. Transactions of the American Society of Civil Engineers, 95(1), 951-960.

Watt, W. E., Chow, K. C. A., Hogg, W. D., \& Lathem, K. W. (1986). A 1-h urban design storm for Canada. Canadian Journal of Civil Engineering, 13(3), 293-300.

Wenzel, H. (1982). Rainfall for urban stormwater design. In: Urban storm water hydrology. Water Resources Monograph 7. Washington DC, USA: American Geophysical Union. 